\title{
EAl Endorsed Transactions

\section{Correlation Analysis of Vital Signs to Monitor Disease Risks in Ubiquitous Healthcare System}

\author{
Hassan Murtaza ${ }^{1}$, Muhammad Azhar Iqbal $^{2, *}$, Qammer H. Abbasi ${ }^{3}$, Sajjad Hussain ${ }^{3}$, Huanlai \\ Xing $^{2}$, Muhammad A. Imran ${ }^{3}$ \\ ${ }^{1}$ Capital University of Science and Technology, Islamabad, Pakistan \\ ${ }^{2}$ School of Information Science and Technology, Southwest Jiaotong University, P. R. China \\ ${ }^{3}$ James Watt School of Engineering, University of Glasgow, United Kingdom
}

\section{Abstract}

Healthcare systems for chronic diseases demand continuous monitoring of physiological parameters or vital signs of the patients' body. Through these vital signs' information, healthcare experts attempt to diagnose the behavior of a disease. Identifying the relationship between these vital signs is still a big question for the research community. We have proposed a sophisticated way to identify the affiliations between vital signs of three specific diseases i.e., Sepsis, Sleep Apnea, and Intradialytic Hypotension (IDH) through Pearson statistical correlation analysis. Vital signs data of about 32 patients were taken for analysis. Experimental results show significant affiliations of vital signs of Sepsis and IDH with average correlation coefficient of 0.9 and 0.58 , respectively. The stability of the mentioned correlation is about $75 \%$ and $90 \%$, respectively.

Received on 03 July 2020; accepted on 23 July 2020; published on 27 July 2020

Keywords: Ubiquitous Healthcare Systems, Vital Sign Analysis, Correlation Analysis

Copyright (C) 2020 Hassan Murtaza et al., licensed to EAI. This is an open access article distributed under the terms of the Creative Commons Attribution license (http://creativecommons.org/licenses/by/3.0/), which permits unlimited use, distribution and reproduction in any medium so long as the original work is properly cited.

doi:10.4108/eai.18-5-2020.165676

\section{Introduction}

Dissemination of chronic diseases and ever-increasing growth of the human population demands the monitoring of an individual's health status through continuous vital sign measurement to prevent fatal disorders. Vital signs measurements of the human body's most fundamental functions (i.e., blood pressure, pulse rate, respiration or breathing rate, and body temperature, etc.) are helpful in the detection and monitoring of medical problems [1] [2]. Through the availability of biomedical sensors and IoT devices, vital signs measurements can be taken anytime from anywhere and ultimately promote the concept of Ubiquitous Healthcare Systems (UHS) [3], telemedicine systems [4], and digital epidemiology [5]. UHS facilitate medical officers and caregivers through continuous observation of patients' vital signs for in-time diagnosis, analysis, and treatment of diseases [6]. The combination of a patient's vital signs is important in disease diagnosis and prognosis because these vital signs are somewhere

*Corresponding author. Email: m.a.iqbal@swjtu.edu.cn related to each other [7]. Medical research has classified vital sign criticality levels for different diseases and the significance of correlation analysis of vital signs has attracted both medical and Big data analysts [8]. In this study, we have proposed a sophisticated way to analyze vital signs relationship for three diseases, Sepsis [9], Sleep Apnea [10], and Intradialytic Hypotension (IDH) [11] [12]. In Sepsis, the body's immune system while fighting an infection goes into overdrive and it has three different levels i.e., normal, severe, and septic shock. Sleep Apnea disease is concerned with one or more pauses in breathing or shallow breaths in sleep, which may cause stroke, hypotension, and heart failure. IDH has been defined as both falls in systolic blood pressure or a decrease in Mean Arterial Pressure (MAP). Concerning Sepsis, Sleep Apnea, and IDH, medical research has already identified significant vital signs and corresponding critical ranges as shown in Table 1.

\section{Literature Review}

Several health monitoring systems [13] [14] [15] have been proposed and developed with an objective of 
Table 1. Selected Diseases and Corresponding Vital Signs' Threshold Values

\begin{tabular}{|c|c|c|c|}
\hline Disease & \multicolumn{3}{|c|}{ Vital Signs } \\
\hline \hline Sepsis & Temperature $>38.3^{\circ} \mathrm{C}$ or $<36^{\circ} \mathrm{C}$ & Pulse $>90 \mathrm{c} / \mathrm{min}$ & Breathing Rate $>20 \mathrm{c} / \mathrm{min}$ \\
Sleep apnea & Pulse $<60$ beats $/ \mathrm{min}$ or $>100$ beats $/ \mathrm{min}$ & SPO $2<90 \%$ & - \\
Hypotension & Systolic $\mathrm{BP}>20 \mathrm{mmHg}$ & Decreasing $\mathrm{MAP}>10 \mathrm{mmHg}$ & - \\
\hline
\end{tabular}

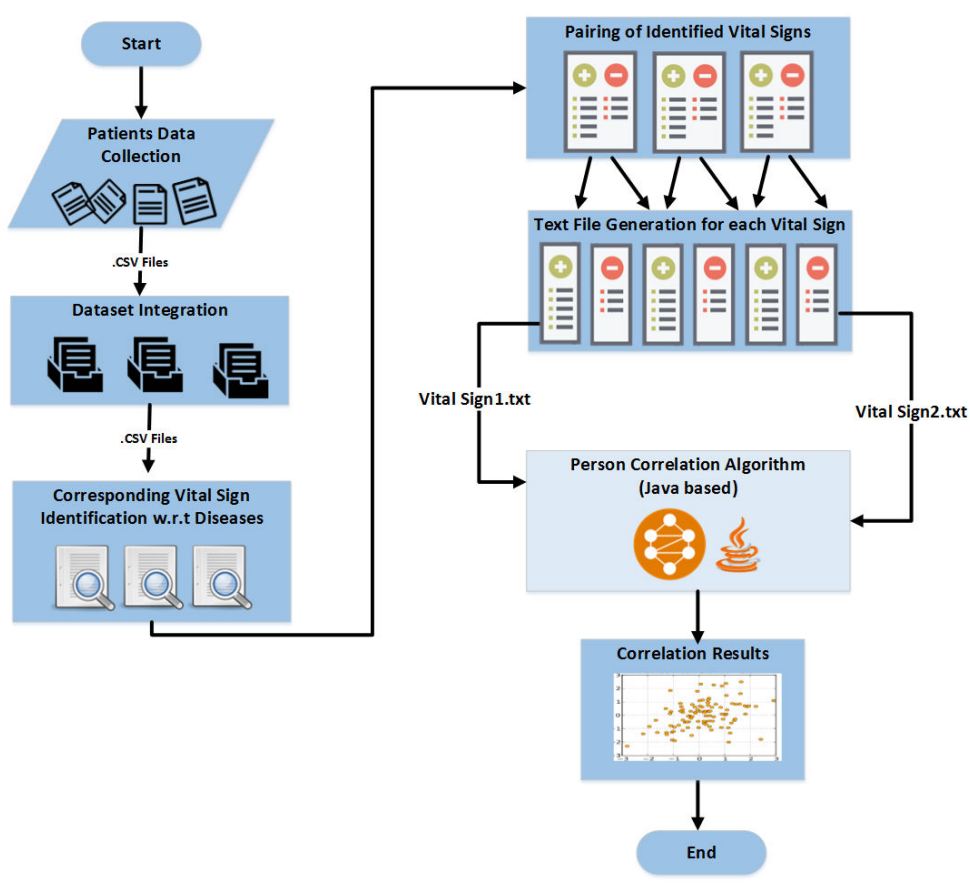

Figure 1. Proposed Work Flowchart

patient/doctor/caregiver assistance through continuous vital sign monitoring. In various studies, it has been observed that a combination of vital signs can be used to assess the criticality level of a disease. Therefore, the correlation and diagnostic value of the combined vital signs have been determined in various studies. In [7], authors have concluded that increased respiratory rate and shock index are highly correlated with disease severity in patients with Sepsis. Thompson et al. [16] have proved that a combination of vital signs can be used to assess the seriousness of infections in children. Authors in [17] demonstrated that vital signs are important to predict serious bacterial infections among children with acute febrile illnesses. Moreover, in this study, the authors suggested raising awareness and knowledge about the importance of measuring vital signs among medical staff. Pereira et al. [18] have investigated correlation for various vital signs (i.e., body temperature, blood pressure, and plasmatic nitric oxide) in septic patients. This study demonstrated that inverse correlation exists between body temperature and nitric oxide in patients with septic shock. In [19], the authors proved the association of the concurrent presence of three critically abnormal vital signs (temperature, systolic blood pressure, oxygen saturation) with very high mortality in hospitals. Besides, authors endorsed that early detection of vital signs' criticality level provides an opportunity to decrease mortality rate. Authors in [20] evaluated whether the prediction in hospitalized patients' clinical deterioration could be realized through vital sign trends. However, the authors concluded by emphasizing on the lack of research in the intermittent monitoring of vital sign trends.

\section{Work Methodology}

To perform correlation analysis on vital signs' of three selected diseases (Sepsis, Sleep Apnea, and IDH), we have acquired vital sign dataset from the University of Queensland [21]. The dataset contains 32 surgical patient cases where patients go through anesthesia at the Royal Adelaide Hospital. For each case, 65 vital signs' parameter samples have been collected for a time interval ranging from $13 \mathrm{~min}$ to $5 \mathrm{~h}$, with a 10-millisecond sampling rate. All cases are made available in scattered CSV files. For vital signs' 
Table 2. Correlation Results of Associated Vital Signs for Sepsis, Sleep Apnea, and IDH

\begin{tabular}{|c|c|c|c|c|c|}
\hline Patient Cases & $\begin{array}{c}\text { Temperature } \\
\& \\
\text { Breathing Rate }\end{array}$ & $\begin{array}{c}\text { Temperature } \\
\& \\
\text { Pulse }\end{array}$ & $\begin{array}{c}\text { Pulse } \\
\& \\
\text { Breathing Rate }\end{array}$ & $\begin{array}{l}\text { Sys BP } \\
\& \\
\text { MAP }\end{array}$ & $\begin{array}{l}\text { Pulse } \\
\& \\
\text { SpO2 }\end{array}$ \\
\hline Case 1 & 0.086289 & 0.198967 & 0.237355 & 0.936186 & 0.180717 \\
\hline Case 2 & $\mathrm{NaN}^{\#}$ & 0.678066 & $\mathrm{NaN}$ & 0.732698 & 0.341122 \\
\hline Case 3 & -0.06172 & 0.630976 & -0.15663 & 0.494545 & 0.0155 \\
\hline Case 4 & 0.146256 & 0.906258 & 0.226634 & 0.918874 & -0.27904 \\
\hline Case 5 & 0.07908 & 0.091976 & 0.258619 & 0.985365 & 0.174049 \\
\hline Case 6 & -0.16414 & 0.82221 & -0.16576 & 0.956374 & -0.01867 \\
\hline Case 7 & -0.18353 & 0.791483 & -0.18002 & 0.903671 & -0.00923 \\
\hline Case 8 & 0.104863 & 0.724837 & -0.01164 & 0.940084 & 0.011936 \\
\hline Case 9 & 0.30642 & 0.716878 & 0.500997 & 0.959157 & -0.12958 \\
\hline Case 10 & -0.36317 & 0.708212 & -0.46756 & 0.976464 & -0.39713 \\
\hline Case 11 & -0.0511 & 0.798113 & -0.08402 & 0.970858 & 0.064615 \\
\hline Case 12 & -0.09302 & 0.730333 & -0.0929 & 0.959795 & 0.075773 \\
\hline Case 13 & 0.050499 & 0.369743 & 0.352568 & 0.990612 & -0.13134 \\
\hline Case 14 & $\mathrm{NaN}$ & 0.928549 & $\mathrm{NaN}$ & 0.775414 & 0.083295 \\
\hline Case 15 & $\mathrm{NaN}$ & 0.334489 & $\mathrm{NaN}$ & 0.903326 & -0.64141 \\
\hline Case 16 & 0.13527 & 0.75297 & 0.294293 & 0.823765 & -0.17975 \\
\hline Case 17 & $\mathrm{NaN}$ & -0.02376 & $\mathrm{NaN}$ & 0.949645 & 0.081921 \\
\hline Case 18 & $\mathrm{NaN}$ & 0.495586 & $\mathrm{NaN}$ & 0.993089 & -0.39638 \\
\hline Case 19 & $\mathrm{NaN}$ & 0.004187 & $\mathrm{NaN}$ & 0.85501 & 0.321408 \\
\hline Case 20 & 0.159129 & 0.705809 & 0.192151 & 0.927507 & -0.01769 \\
\hline Case 21 & 0.042119 & 0.920661 & 0.050578 & 0.928075 & -0.06405 \\
\hline Case 22 & -0.09787 & 0.841802 & -0.07986 & 0.683595 & 0.139722 \\
\hline Case 23 & -0.21756 & 0.363275 & 0.140553 & 0.978274 & -0.11101 \\
\hline Case 24 & -0.59494 & 0.767755 & -0.64434 & 0.939129 & -0.34544 \\
\hline Case 25 & $\mathrm{NaN}$ & 0.440382 & $\mathrm{NaN}$ & 0.75032 & -0.35877 \\
\hline Case 26 & -0.0287 & 0.734474 & 0.002419 & 0.914842 & -0.2557 \\
\hline Case 27 & -0.11765 & 0.634821 & 0.118373 & 0.853356 & -0.18995 \\
\hline Case 28 & 0.104937 & 0.620229 & 0.062052 & 0.969606 & 0.124855 \\
\hline Case 29 & 0.031549 & 0.766763 & 0.016561 & 0.97496 & -0.0445 \\
\hline Case 30 & -0.09056 & 0.31498 & -0.19463 & 0.946691 & -0.13697 \\
\hline Case 31 & -0.19697 & 0.578791 & -0.27978 & 0.956476 & 0.053384 \\
\hline Case 32 & -0.19168 & 0.803928 & -0.25545 & 0.950706 & -0.08212 \\
\hline
\end{tabular}

\#: NaN - Not a Number

correlation analysis, we have considered six vital signs (i.e., Temperature, Oxygen Saturation (SpO2), Breathing Rate, Pulse rate, Systolic BP and MAP), which are concerned to our selected set of diseases. Our research methodology consists of five major phases (i.e., data set integration, vital sign classification, dataset filtering, file generation, and implementation of Pearson correlation algorithm [22]) as shown in Figure 1. Details of these five steps are given below:

\subsection{Dataset Integration}

In the first phase, we have automated the process of integrating multiple available files into a single combined CSV file. The integrated file contains all significant and non-significant vital sign information which ultimately required for corresponding vital signs' identification and filtration for selected diseases.

\subsection{Vital Sign Identification}

For monitored diseases, the study of Kateretse and Huh [23] helped us to perform the identification of vital sign pairs for correlation analysis. For each selected disease, identified pairs of corresponding vital signs are mentioned below:

Sepsis:

- Temperature/Pulse

- Pulse/Breathing Rate

- Breathing Rate/Temperature

Sleep Apnea:

- Pulse/SpO2

Hypotension:

- Systolic BP/MAP 


\subsection{Dataset Filtration and File Generation}

After corresponding vital sign pairs identification, an automated dataset filtration process considered pairs of identified vital signs (only for which values exist in dataset) to be copied in a separate text file. These generated text files used as input for the Pearson algorithm to perform correlation analysis. In this phase, considering two files for each vital sign pair, 320 files have been generated for all cases.

\subsection{Implementation of Pearson Algorithm}

Pearson Algorithm is a standard correlation analysis algorithm [22], which informs about the dependence of two variables. Value of correlation varies from -1 to +1 ; where $+1,-1$, and 0 values represent total positive, total negative and 0 correlation, respectively. Generated vital sign pair files are given as input to our Javabased implemented Pearson algorithm to get coefficient results.

\section{Results and Discussion}

Following the workflow shown in Figure 1, for statistical analysis of vital signs of above-mentioned diseases, we have computed five major vital signs correlations:

- Temperature and Breathing Rate (for Sepsis disease)

- Temperature and Pulse (for Sepsis disease)

- Pulse and Breathing Rate (for Sepsis disease)

- Systolic BP and Decreasing Mean BP (for Sleep Apnea disease)

- Pulse and SpO2 (for IDH disease)

Mentioned correlation coefficients are computed for all 32 cases of the provided dataset. Table 2 shows the correlation results of the above mentioned vital signs.

\section{Results}

From Table 2, it becomes obvious that for Sepsis and IDH diseases, there exists significant correlation in their few vital signs parameters. Concerning Sepsis disease, $60 \%$ average correlation appeared for Temperature and Pulse Rate. However, for other two vital sign correlations (i.e., Pulse/Breathing Rate and Temperature/Breathing Rate) did not provide significant results. Figures 2, 3, and 4 show the statistical plots of each correlation for Sepsis, Sleep Apnea, and IDH, respectively. In Figures 2(a) and 2(c), there exist random behavior of correlation results for each case but for both vital signs' correlation (Pulse/Breathing Rate and Temperature/Breathing

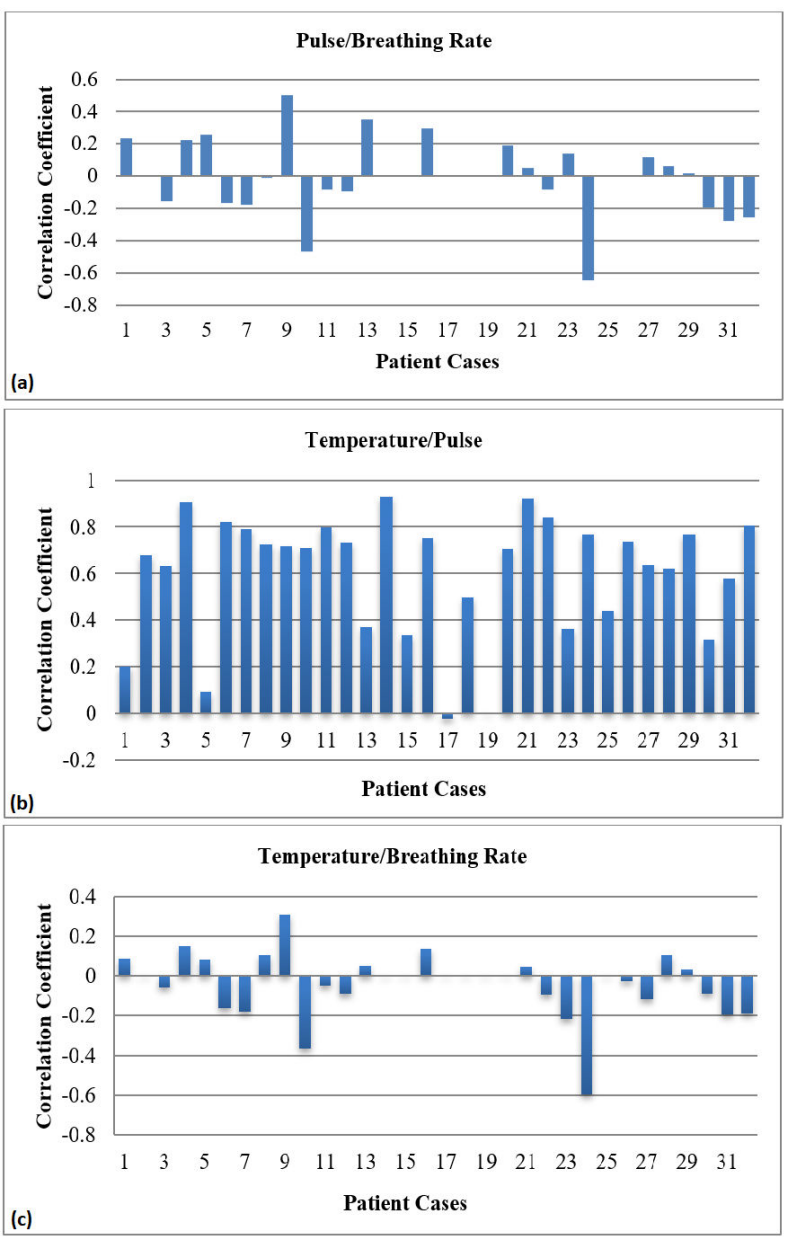

Figure 2. (a), (b), (c):Correlation Results of Vital Signs for Sepsis Disease

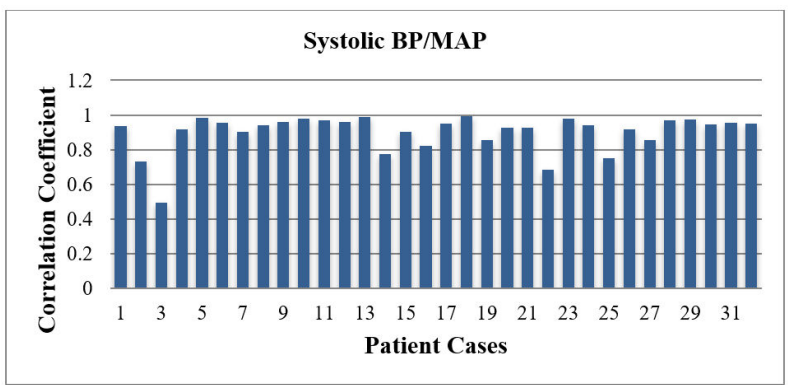

Figure 3. Correlation Results of vital signs for IDH

Rate) correlation direction (Positive/Negative or no correlation) for individual cases is $90 \%$ identical. Therefore, it is concluded that the trend for both correlation results (Figure 2(a) and Figure 2(c)) is almost the same for individual patient cases. The common factor for both scenarios is Breathing Rate which might participate to improve that trend. In Figure 2(a) about 11 cases (34.5\%) have negative 


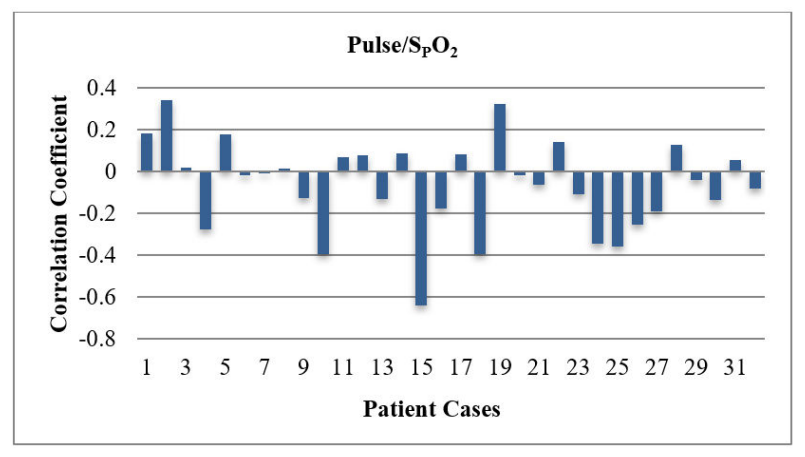

Figure 4. Correlation Results of Vital Signs for Sleep Apnea

correlation, 10 cases $(31 \%)$ have positive correlation and 11 cases $(34.5 \%)$ cases have no correlation. In Figure 2(c) about 12 cases $(37.5 \%)$ have negative correlation, 7 cases $(22 \%)$ have positive correlation and 13 cases $(40.5 \%)$ cases have no correlation.

In Figure 2(b) and Figure 3, there exists significant correlation in Temperature/Pulse and Systolic $\mathrm{BP} / \mathrm{MAP}$, respectively. Correlation results for most of the cases are appeared positive as shown in Figure 2(b). Approximately, 30 cases (94\%) have positive correlation and 2 cases $(6 \%)$ have no correlation. About $90 \%$ average positive correlation exists for Systolic BP and MAP (shown in Figure 3), which is associated with IDH disease. From Figure 3, it becomes obvious that in all cases correlation results appear positive. About $60 \%$ (correlation coefficient $=0.6$ ) average positive correlation exists for Temperature and Pulse (shown in Figure 2(b)), which are associated with Sepsis disease.

For Sleep Apnea, no significant correlation results appeared (for Pulse and SpO2) as shown in Figure 4. In Figure 4, for most of the cases, correlation results appeared negative. About 15 cases (47\%) have negative correlation, 11 cases $(34 \%)$ have positive correlation and 6 cases $(19 \%)$ cases have no correlation.

Figure 5 presenting the scatter plot of abovementioned correlation results for all 32 cases. Figure 5 also provides the linearity trend line of each correlation results. According to the definition of linearity, as much as the linearity trend line will be stable on the $\mathrm{x}$-axis, it will show correlation results' stability. The linear trend line for Temperature/Pulse correlation is very stable as shown in Figure 5, which concluded that correlation result precision is above $90 \%$. For the Systolic BP/MAP correlation, the linearity trend is about $75 \%$. Moreover, the other three trend lines are not considerable because of their insignificant correlation results.

\section{Conclusion}

In this paper, we have analyzed the correlation among various vital signs for the purpose of monitoring the associated disease risks. Contrary to most of the existing schemes, we have used a real-life dataset of 32 patients for the analysis of three specific diseases that are found to be as common ailments in hospitalized patients. The analysis presented in this paper is helpful in the process to predict the disease pattern and patients' health condition. Our results show that there exists significant correlation among the studied vital signs, and we can use this correlation to predict the behavior of vital signs that may be unavailable in a specific scenario due to lack of resources. In the future, we aim to extend this work by applying machine learning techniques to find useful correlation among vital signs to predict risks of different diseases. Moreover, we intend to build an early alarm system using these predictions that can give some extra critical time to the doctors to react and save patients' lives.

\section{References}

[1] Chakraborty, C., Gupta, B. and Ghosh, S.K. (2013) A review on telemedicine-based wban framework for patient monitoring. Telemedicine and e-Health 19(8): 619626.

[2] Yilmaz, T., Foster, R. and Hao, Y. (2010) Detecting vital signs with wearable wireless sensors. Sensors 10(12): 10837-10862.

[3] Otto, C., Milenkovic, A., Sanders, C. and Jovanov, E. (2006) System architecture of a wireless body area sensor network for ubiquitous health monitoring. Journal of mobile multimedia 1(4): 307-326.

[4] Salem, O., Liu, Y., Mehaoua, A. and Boutaba, R. (2014) Online anomaly detection in wireless body area networks for reliable healthcare monitoring. IEEE journal of biomedical and health informatics 18(5): 15411551.

[5] Salathe, M., Bengtsson, L., Bodnar, T.J., Brewer, D.D., Brownstein, J.S., Buckee, C., Campbell, E.M. et al. (2012) Digital epidemiology. PLoS Comput Biol 8(7): e1002616.

[6] Murtaza, H., Iqbal, M.A., Islam, M.A. and Aleem, M. (2018) Content-aware reliable and energy-efficient mac (care-mac) protocol for wireless body area networks. Adhoc E Sensor Wireless Networks 41.

[7] Kenzaka, T., Okayama, M., Kuroki, S., Fukui, M., Yahata, S., Hayashi, H., Kitao, A. et al. (2012) Importance of vital signs to the early diagnosis and severity of sepsis: association between vital signs and sequential organ failure assessment score in patients with sepsis. Internal Medicine 51(8): 871-876.

[8] Wang, L. and Alexander, C.A. (2020) Big data analytics in medical engineering and healthcare: methods, advances and challenges. Journal of Medical Engineering E Technology : 1-17.

[9] Vincent, J.L., Moreno, R., Takala, J., Willatts, S., De Mendonça, A., Bruining, H., Reinhart, C. et al. (1996), The sofa (sepsis-related organ failure assessment) score to describe organ dysfunction/failure.

[10] Suzuki, T., Kameyama, K.I., Inoko, Y. and Tamura, T. (2010) Development of a sleep apnea event detection 


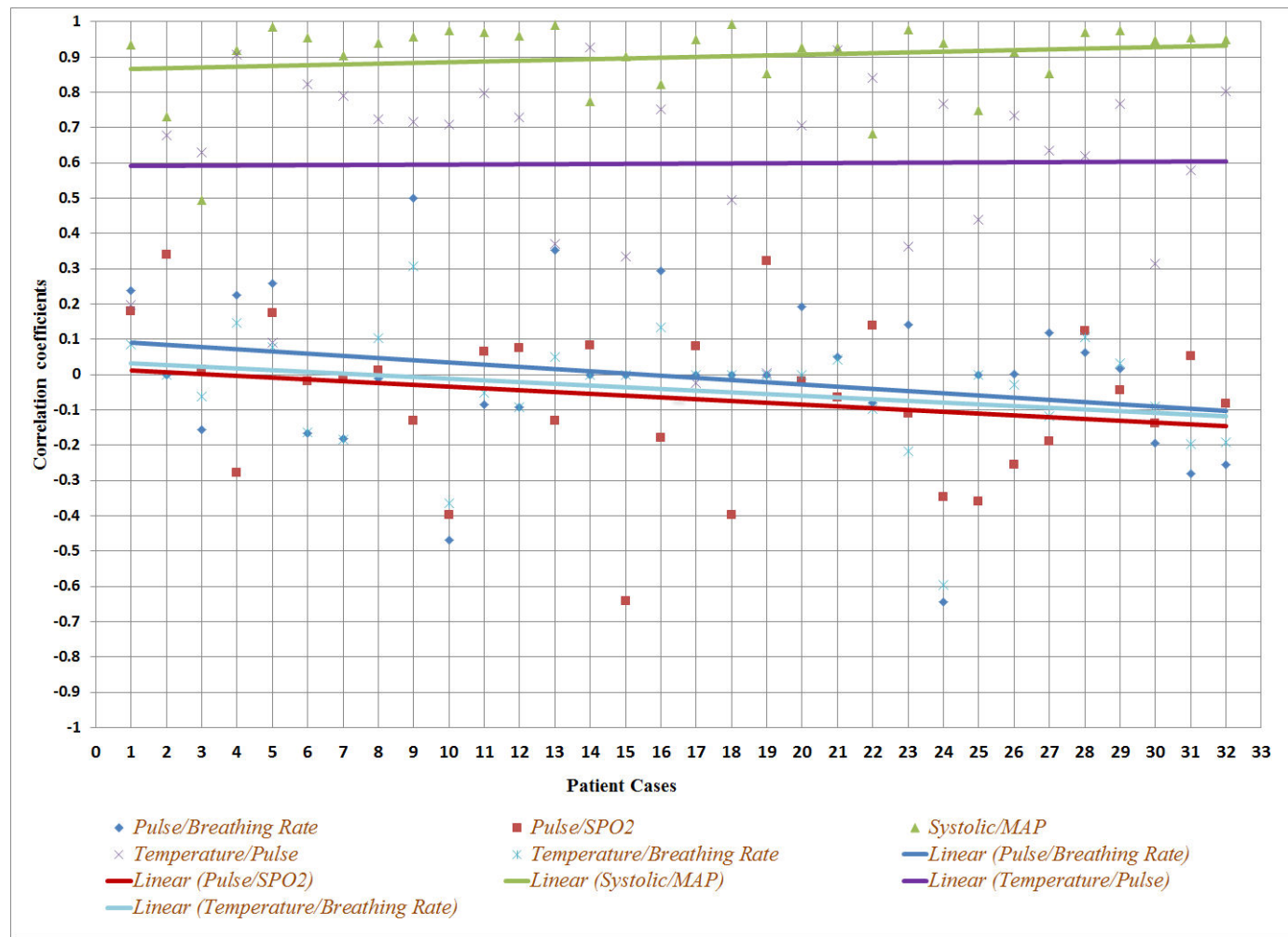

Figure 5. Linearity of Correlation Results

method using photoplethysmography. In 2010 Annual International Conference of the IEEE Engineering in Medicine and Biology (IEEE): 5258-5261.

[11] Bradshaw, W. (2014) Intradialytic hypotension: a literature review. Renal Society of Australasia Journal 10(1).

[12] Wu, Y.C., LaI, T.Y., Lin, T.S., Wang, J.Y., ShieH, J.C., JiAnG, J.A., Chang, W.D. et al. (2009) A wsn-based wireless monitoring system for intradialytic hypotension of dialysis patients. In SENSORS, 2009 IEEE (IEEE): 1959-1962.

[13] Nayan, N.A. and Azami, F.A. (2014) Continuous vital signs monitoring using smartphones. i-IDeA, International Innovation, Design and Articulation, India .

[14] Shahriyar, R., Bari, M.F., Kundu, G., Ahamed, S.I. and AквAR, M.M. (2009) Intelligent mobile health monitoring system (imhms). In International Conference on Electronic Healthcare (Springer): 5-12.

[15] Abdullah, A., Ismael, A., Rashid, A., Abou-ElNour, A. and TARique, M. (2015) Real time wireless health monitoring application using mobile devices. International Journal of Computer Networks \& Communications (IJCNC) 7(3): 13-30.

[16] Thompson, M., Coad, N., Harnden, A., Mayon-White, R., Perera, R. and Mant, D. (2009) How well do vital signs identify children with serious infections in paediatric emergency care? Archives of disease in childhood 94(11): 888-893.

[17] Salah, E.T., Ahmed, E., Elhussien, M. and Salah, T. (2014) The use of vital signs as predictors for serious bacterial infections in children with acute febrile illness in a pediatric emergency setting in sudan. Sudanese journal of paediatrics 14(2): 35.

[18] Pereira, F.H., Batalhão, M.E. and Cárnio, E.C. (2014) Correlation between body temperature, blood pressure and plasmatic nitric oxide in septic patients. Revista latino-americana de enfermagem 22(1): 123-128.

[19] Bleyer, A.J., Vidya, S., Russell, G.B., Jones, C.M., Sujata, L., Daeihagh, P. and Hire, D. (2011) Longitudinal analysis of one million vital signs in patients in an academic medical center. Resuscitation 82(11): 13871392.

[20] Brekke, I.J., Puntervoll, L.H., Pedersen, P.B., Kellett, J. and Brabrand, M. (2019) The value of vital sign trends in predicting and monitoring clinical deterioration: A systematic review. PloS one 14(1): e0210875.

[21] Liu, D., Görges, M. and Jenkins, S.A. (2012) University of queensland vital signs dataset: development of an accessible repository of anesthesia patient monitoring data for research. Anesthesia \& Analgesia 114(3): 584589.

[22] Cohen, J., Cohen, P., West, S.G. and Aiken, L.S. (2013) Applied multiple regression/correlation analysis for the behavioral sciences (Routledge).

[23] Kateretse, C. and Huh, E.N. (2011) Efficient data transmission scheme for ubiquitous healthcare using wireless body area networks. In International Conference on Software Engineering and Computer Systems (Springer): 324-335. 